\title{
The Conformational Sensitivity of Iterative Stockholder Partitioning Schemes
}

\author{
T. Verstraelen ${ }^{\mathrm{a}, *}$, P. W. Ayers ${ }^{\mathrm{b}}$, V. Van Speybroeck ${ }^{\mathrm{a}}$, M. Waroquier $^{\mathrm{a}}$ \\ ${ }^{a}$ Center For Molecular Modeling, Ghent University, Technologiepark 903, 9050 Zwijnaarde, Belgium (Member of \\ the QCMM Ghent-Brussels Alliance Group) \\ ${ }^{b}$ McMaster University, Department of Chemistry, Hamilton, ON L8S 4M1, Canada
}

\begin{abstract}
Chemical interpretation and empirical modeling of partial charges requires a robust partitioning scheme to derive these charges from the molecular electronic density. The degree of undesirable conformational sensitivity is assessed for three iterative stockholder partitioning schemes: Hirshfeld-I (HI), Iterative Stockholder Analysis (ISA) and a new Gaussian ISA variant (GISA). GISA has fewer degrees of freedom than ISA and enforces monotonically decaying pro-atoms. These improvements accelerate the converge of GISA as compared to ISA. However, the conformational sensitivity of the charges does not decrease and is still large compared to HI.
\end{abstract}

Keywords: population analysis, stockholder partitioning, robustness, transferability, locality

\section{Introduction}

A pervasive characteristic of chemistry is that the properties of a molecule can be explained in terms of its constituent atoms and functional groups. This contrasts sharply with theoretical and computational picutre of a molecule as a system of nuclei and electrons. With the quantum mechanical description of the electronic many-body systen, one can model molecular properties without ever assigning electrons to certain atoms. It is even far from trivial to partition the electronic density (or wavefunction) into atomic contributions based on theoretical arguments [1, 2]. Despite these difficulties, partitioning schemes remain essential to the theoretical foundation of the plethora of rules in chemistry that connect the behavior of a molecule with the properties of its atoms.

The partitioning of the molecular electronic structure in atomic contributions was pioneered by

\footnotetext{
*Corresponding author

Email addresses: Toon.Verstraelen@UGent.be (T. Verstraelen), Michel.Waroquier@UGent.be (M. Waroquier)
} 
Mulliken [3]. Originally, the Mulliken scheme was introduced to estimate atomic partial charges, but it can also be used to compute any atom-in-molecule (AIM) property. One of the critical weaknesses of Mulliken's scheme is the lack of robustness, i.e. the AIM quantities are very sensitive to the choice of basis set for the expansion of the wavefunction and to conformational changes [46]. The Natural Population Analysis (NPA) is a popular variant of Mulliken's scheme that yields more robust AIM properties. [7]

In this work, robustness refers not just to the stability of an AIM scheme with respect to numerical round-off errors, but also to AIM properties that are not sensitive to other computational parameters like the choice of the basis set, small conformational changes, choice of integration grids, and so on. Robustness is a desirable property of an AIM scheme because it is a requirement for the transferability (between different molecules) of AIM quantities of similar atoms or functional groups. This is of critical importance for the chemical interpretation of AIM properties or for the derivation of transferable force field parameters. For example, the quantum theory of atoms in molecules (QTAIM) scheme proposed by Bader [8] yields transferable AIM properties [2, 9]. On the other hand, charges fitted to reproduce the molecular ESP are traditionally not robust. Even with the aid of common regularization techniques, e.g. such as in the RESP scheme [10], ESP fitted charges exhibit an erratic dependence on the molecular internal coordinates [6].

Several studies have shown that the Hirshfeld-I (HI) scheme [11] is robust with respect to conformational changes and the choice of basis set. $[6,12,13]$ It is also known that, for organic molecules, HI atomic charges accurately reproduce the ESP surrounding the molecule $[14,15]$. This is a distinct advantage of HI over QTAIM and implies that HI charges are both useful for a direct chemical interpretation as well as for the development of force field models. Unfortunately, a recent study revealed that HI charges severely overestimate molecular dipoles of isolated inorganic clusters [16], showing that the accurate reproduction of the molecular ESP is not a universal property of the HI scheme. Because of this deficiency, it is clear that the Hirshfeld-I scheme can be improved. In order to do so, a profound understanding of the origins of the robustness and ESP accuracy of AIM charges is mandatory. In this letter we analyze the robustness of several AIM schemes that are based on the stockholder principle, i.e. Hirshfeld-I (HI) [11], Iterative Stockholder Analysis (ISA) [17], and a new variant of ISA. This analysis is not only relevant for for the development of improved partitioning schemes, but also for dispersion corrections in density functional theory that are based on Hirshfeld partitioning [18-20]. In order to obtain a smooth dispersion correction 
to the potential energy surface, the underlying partitioning scheme must be robust.

It was already suggested earlier that the ISA scheme could be problematic in terms of robustness because it involves the optimization of a large number of degrees of freedom [21]. As an attempt to design a more robust alternative, the Gaussian ISA variant (with fewer degrees of freedom) is proposed in this work. The robustness of each scheme will be assessed by studying the conformational sensitivity of selected atomic charges in the set of 103 randomized penta-alanine conformers. This set was already used successfully in earlier work to compare the conformational robustness of other population analysis schemes and two empirical charge models [6].

The structure of this letter is as follows. The next section reviews the original (non-iterative) Hirshfeld, HI, and ISA schemes and introduces the Gaussian ISA variant. The third section describes the computational details. The fourth section describes the assessment protocol used to compare the robustness of the AIM schemes. Section five discusses the results of the assessment. The last section summarizes the main conclusions of this work.

\section{Stockholder Partitioning}

The general principle of stockholder partitioning is that one proposes pro-atomic densities for all atoms in a molecule, which are then used to define atomic weight functions.[22, 23] Each atomic weight function is the ratio of the corresponding pro-atom and the sum of all pro-atoms:

$$
w_{A}(\mathbf{r})=\frac{\rho_{A}^{\mathrm{pro}}(\mathbf{r})}{\sum_{B} \rho_{B}^{\mathrm{pro}}(\mathbf{r})} .
$$

The sum of these weight functions is unity by construction. The AIM densities are then defined as follows:

$$
\rho_{A}(\mathbf{r})=w_{A}(\mathbf{r}) \rho_{\mathrm{mol}}(\mathbf{r})
$$

The atomic charge can be derived from the AIM density:

$$
q_{A}=Z_{A}-N_{A} \quad \text { with } \quad N_{A}=\int \rho_{A}(\mathbf{r}) d \mathbf{r}
$$

where $N_{A}$ is the atomic population of atom $A$. Hirshfeld [22], Hirshfeld-I [11], Iterative Stockholder Analysis [17] and the new scheme proposed below only differ in the definition of the pro-atomic densities. One can show, based on arguments from information theory, that the stockholder approach partitions the molecule into atomic densities that are maximally similar to the pro-atoms $[24,25]$. 
In the original method proposed by Hirshfeld $(\mathrm{H})$ [22], the pro-atoms are the spherically averaged densities of neutral isolated atoms, centered at the corresponding nuclei in the molecule:

$$
\rho_{A}^{\mathrm{pro}, \mathrm{H}}(\mathbf{r})=\left\langle\rho_{A}^{0}\right\rangle\left(\left|\mathbf{r}-\mathbf{r}_{A}\right|\right)
$$

where the angle brackets are used to denote the spherical average. It was found that Hirshfeld charges are always relatively small in absolute value [26], which is not surprising given that the Hirshfeld AIMs are maximally similar to neutral atoms.

The choice for neutral atoms in the Hirshfeld scheme is in principle arbitrary [26]. One could as well use spherically averaged densities of isolated ions. Hirshfeld-I [11] circumvents this ambiguity by imposing a self-consistency between the population of the pro-atom and the atom in a molecule. A Hirshfeld-I pro-atom for a given population, $N_{A}$, is constructed as a linear combination between the spherically averaged densities of the nearest isolated ions. With the aid of the floor $\left(\left\lfloor N_{A}\right\rfloor\right)$ and ceiling $\left(\left\lceil N_{A}\right\rceil\right)$ functions, this can be written as:

$$
\begin{aligned}
\rho_{A}^{\mathrm{pro}, \mathrm{HI}}\left(\mathbf{r}, N_{A}\right) & =\left(\left\lceil N_{A}\right\rceil-N_{A}\right)\left\langle\rho_{A}^{0}\right\rangle\left(\left|\mathbf{r}-\mathbf{r}_{A}\right| ;\left\lfloor N_{A}\right\rfloor\right) \\
& +\left(N_{A}-\left\lfloor N_{A}\right\rfloor\right)\left\langle\rho_{A}^{0}\right\rangle\left(\left|\mathbf{r}-\mathbf{r}_{A}\right| ;\left\lceil N_{A}\right\rceil\right)
\end{aligned}
$$

Because the Hirshfeld-I atomic populations are not known a priori, one first uses an initial guess, e.g. the Hirshfeld populations, to construct a first approximation of the Hirshfeld-I pro-atoms. In a second iteration, these pro-atoms are used to recompute the atomic populations and to update the approximation of the Hirshfeld-I pro-atoms. This iterative procedure is continued until the Hirshfeld-I populations converge, which typically takes about 25 iterations in small molecules. Unlike the Hirshfeld scheme, the Hirshfeld-I scheme contains an unknown parameter per atom $\left(N_{A}\right)$ that must be converged iteratively.

The iterative stockholder analysis (ISA) [17] makes no a-priori assumption on the form of the pro-atom, except that it must be spherically symmetric. In ISA, the pro-atom must coincide with the spherical average of the AIM, which can be written as follows:

$$
\rho_{A}^{\mathrm{pro}, \mathrm{ISA}}\left(\mathbf{r}, \rho_{A}\right)=\left\langle\rho_{A}\right\rangle\left(\left|\mathbf{r}-\mathbf{r}_{A}\right|\right)
$$

In practice, this relation is imposed for every atom on a limited set of radial grid points. For all other distances from the nucleus, the pro-atom is estimated with a suitable interpolation scheme. In analogy with the Hirshfeld-I scheme, one first constructs an initial guess for the ISA pro-atomic densities and one then updates them iteratively until they converge. The number of unknowns 
that must be determined self-consistently equals the number of atoms times the number of radial grid points. This implies that the number of degrees of freedom is much higher than in the HI scheme. The main advantage of ISA, both conceptually and practically, is that the method does not rely on atomic reference densities that must be computed a priori. The ISA scheme only uses information extracted from the molecular electron density.

It was found that-for some atoms in some molecules-the density of the ISA pro-atom does not decay monotonically [21], which is a counter-intuitive result [27-29]. We propose a simple modification of the ISA scheme, namely Gaussian ISA (GISA), in which the monotonic decay of the pro-atomic density is enforced with an expansion in normalized Gaussian s-type density basis functions:

$$
\rho_{A}^{\mathrm{pro}, \mathrm{GISA}}\left(\mathbf{r}, \rho_{A}\right)=\sum_{k} D_{A, k}\left(\frac{\alpha_{A, k}}{\pi}\right)^{\frac{3}{2}} \exp \left(-\alpha_{A, k}\left|\mathbf{r}-\mathbf{r}_{A}\right|^{2}\right)
$$

where the expansion coefficients, $D_{k}$, are estimated from a least-squares fit on the spherical average of the AIM density:

$$
\sum_{k} D_{A, k}\left(\frac{\alpha_{A, k}}{\pi}\right)^{\frac{3}{2}} \exp \left(-\alpha_{A, k}\left|\mathbf{r}-\mathbf{r}_{A}\right|^{2}\right) \approx\left\langle\rho_{A}\right\rangle\left(\left|\mathbf{r}-\mathbf{r}_{A}\right|\right)
$$

By imposing constraints $D_{A, k} \geq 0$ and $\sum_{k} D_{A, k}=N_{A}$ during the fit, the pro-atom is guaranteed to be positive, monotonically decaying and consistent with the AIM population. Again, just as in Hirshfeld-I and ISA, the expansion coefficients must be determined iteratively. The choice of the exponents, $\alpha_{k, A}$, is similar to the choice of the grid points in the ISA scheme. However, one can reasonably approximate the spherical average of an AIM density with just a few basis functions (about three to four per shell), while the number of radial grid points in ISA is typically an order of magnitude larger. Due to the lower number of variables per pro-atom, one expects the GISA scheme to be more conformationally robust than the ISA scheme.

\section{Computational details}

The robustness of three partitioning schemes was tested: Hirshfeld-I (HI), Iterative Stockholder Analysis (ISA) and the newly proposed Gaussian Iterative Stockholder Analysis (GISA). For this

purpose, atomic charges were computed with these three schemes for the set of 103 randomly generated terminally blocked penta-alanine conformers that was introduced in an earlier assessment study [6]. All geometries were optimized with Gaussian 09 [30] at the PBE0/6-311+G(d,p) [3133] level of theory. PBE0 properly describes the internal hydrogen bonds in the penta-alanine 
structures at a modest computational cost, also when larger basis sets are used. Diffuse functions were used to assure that atoms with negative partial charges are well-described. The polarization functions assure that the DFT computations properly account for internal polarization effects, e.g. due to the formation of hydrogen bonds. We also performed the same computations on a single neopentane molecule, to compare our results for GISA with an earlier analysis of the ISA scheme $[21]$.

The three partitioning schemes were applied to the electron densities of the optimized pentaalanine structures. The numerical integrations required for the partitioning were carried out on spherical atom-centered grids. The angular integration was carried out on a Lebedev grid with 266 points [34]. The radial grid consisted of 200 points that are equidistant on a logarithmic scale,

ranging from 0.0002 to $20.0 \AA$. Due to the locality of the atomic weight functions, each integral can be accurately evaluated on the spherical grid of the corresponding atom. All the partitioning computations were performed with our in-house code HiPart. (See http://molmod.ugent.be/code)

The database of isolated pro-atomic densities for the Hirshfeld-I method were also computed at the PBE0/6-311+G(d,p) level of theory. For hydrogen, only the neutral atom was considered. For all other elements, the cation, the neutral atom and the anion were computed. The computed densities of the isolated cations, neutral atoms and anions were also used to fit the exponents for the GISA method. For each element, the exponents of a compact Gaussian s-type density basis set were optimized, such that the basis can accurately represent all the associated pro-atomic densities. The exponents used in this work, are given in Table 1.

\section{Assessment protocol}

The standard deviations of the atomic charges (over all penta-alanine conformers) in the central residue, see Fig. 1, were used to investigate the robustness of the AIM schemes. Because pentaalanine is very flexible, the central residue is exposed to diverse orientations and positions of the other residues in each conformer. Furthermore, penta-alanine is well-behaved in terms of polarizability: it does not have large delocalized $\pi$-bonds or charge-transfer states. Therefore, a robust partitioning scheme should not exhibit a large sensitivity of the atomic charges to conformational changes and one can use the spread on each charge as an inverse indicator for the robustness of an AIM scheme with respect to conformational changes.

In order to reveal the origin of the conformational fluctations of a given atomic charge, the 
contributions to the atomic population were analyzed in more detail. Consider the expression for the population of atom $A$ in spherical coordinates (with the nucleus of atom $A$ at the origin):

$$
\begin{aligned}
N_{A} & =\int n_{A}(r) d r \\
n_{A}(r) & =r^{2} \int_{0}^{\pi} \sin \theta \int_{0}^{2 \pi} \rho_{A}(r, \theta, \phi) d \theta d \phi
\end{aligned}
$$

$n_{A}(r) d r$ represents the contribution to the atomic population of atom $A$ in the interval $[r, r+d r]$. Hence, the function $n_{A}(r)$ can be used to analyze at which distances from the nucleus density is assigned to atom $A$. For each AIM scheme, the average and the standard deviation of $n_{A}(r)$ over all conformers was computed for the atoms in the central residue.

\section{Results and discussion}

Before discussing the results for the penta-alanine conformers, we first consider the neopentane molecule. Fig. 2 displays the pro-atoms and spherically averaged AIMs for the central carbon atom. The four other carbon atoms (and similarly for all hydrogen atoms) lie at the same distance from this central carbon, which is known to be a pathological situation for the ISA scheme. As was reported earlier [21], the ISA pro-atom, which coincides with the spherical average of the AIM, has several minima and maxima. The first minimum lies at the $\mathrm{C}-\mathrm{C}$ bond length $\left(d_{1}\right)$ and the second minimum coincides with the distance between two methyl carbons $\left(d_{3}\right)$. These correlations clearly show that the ISA AIM for the central carbon atom is affected by its molecular environment. Hirshfeld-I and GISA successfully enforce a sensible behavior on the pro-atoms.

Fig. 2 also shows that the GISA pro-atom does not deviate very much from the corresponding spherically averaged AIM. Hence, the GISA pro-atom almost fulfills the conditions of the ISA pro-atom, yet they are very different. This has quite severe implications on the robustness of the ISA scheme. Although it is shown that the ISA partitioning is uniquely defined [21, 35], i.e. that it corresponds to the minimization of a convex information loss function, this example reveals that the information loss minimum can be nearly degenerate. Hence, the ISA pro-atoms become very sensitive to small details in the molecular electron density, the displacement of nearby atoms, the choice of integration grids, and so on. For the same reason, it is also to be expected that the convergence of ISA will be slower than that of HI or GISA. These signs of non-robustness will carefully monitored in the assessment based on the penta-alanines below. 
The first important result of the penta-alanine assessment is that the convergence of the ISA charges failed for 22 out of 103 conformers, i.e. the difference between the ISA pro-atoms in subsequent iterations did not sufficiently decrease after 5000 iterations. All computations of the HI and GISA charges converged properly. Therefore, the remainder of the analysis includes only the 81 structures for which the ISA partitioning was successful. The average number of iterations needed to converge the ISA charges was 1147, while HI and GISA converged much faster, with on average 43 and 141 iterations, respectively. This indicates again that the ISA partitioning is not robust.

Fig. 3 contains the histograms of the HI, ISA and GISA charges for the selected atoms (see Fig. 1) in the 81 penta-alanine conformers. The standard deviations of the histograms are compared in table 2. The obvious result is that the spread on the HI charges is systematically smaller than the spread on the ISA and GISA charges. In spite of our attempt to construct a more robust ISA variant, with fewer degrees of freedom, GISA charges have a conformational sensitivity that is on par with ISA.

In order to gain more insight in the origin of the differences in robustness, Fig. 4 shows, for the selected atoms in the residue, the average and the standard deviation of the integrand, $n_{A}(r)$, over the 81 conformers. The standard deviation is relatively small compared to the average and is magnified by a factor of 400 for the sake of clarity. The figure shows that, on average, the integrands are very similar in all three schemes. The differences between the schemes are most prominent in the fluctuations of the integrands with respect to the average. Consider for example atom C:28. For this atom, the spread on the ISA charge is mainly due to density changes outside the valence shell, i.e. due to polarization and displacement of neighboring atoms. We found the same trend for all GISA and ISA results of other atoms in penta-alanine. The fluctuations on the integrand in the case of Hirshfeld-I are much smaller, and often (but not always) localized inside the valence shell. This suggests that locality of the fluctuations of AIM densities is a sufficient (but not a necessary) condition for the robustness of the charges.

One can try to improve the robustness of the GISA scheme by discarding the most diffuse s-type basis functions, i.e. the smallest exponents in Table 1. Such an ad hoc solution is similar to avoiding diffuse orbital basis functions in order to obtain robust Mulliken charges. Such attempts to repair GISA actually show the real weakness of the method: one is forced to find a trade-off between the accuracy of the s-type basis for the pro-atoms and the robustness of the partitioning 
scheme. There is no unique or objective criterion to balance out both requirements, which means that the GISA scheme is inherently ambiguous.

The relation between locality and robustness can be applied to understand the properties of other partitioning schemes, which are not necessarily based on the Stockholder principle. Table 2 compares the standard deviation of the Hirshfeld-I charges with QTAIM and RESP charges for the 81 penta-alanine conformers. The QTAIM method is known to yield transferable AIM properties, which corresponds well with the locality of the AIMs. In terms of conformational robustness, it only slightly worse than Hirshfeld-I. ESP-fitting schemes typically predict charges that are highly sensitive to conformational changes, as is also the case in this assessment. One could interpret ESP-fitting as the partitioning of the electronic Hartree potential outside the molecule into atomic contributions of the form $1 /\left|\mathbf{r}-\mathbf{r}_{A}\right|$. These atomic contributions are extremely non-local, which corresponds well with the lack of robustness of ESP-fitted charges.

\section{Conclusions}

The Gaussian Iterative Stockholder Analysis (GISA) is introduced as a more robust alternative for the Iterative Stockholder Analysis (ISA). In the GISA scheme, pro-atoms are expanded in stype Gaussian basis functions with positive coefficients. This new method has two main advantages over the original ISA: (i) fewer variables need to be converged for the partitioning of the molecular electron density and (ii) the pro-atoms are always monotonically decaying functions. The only additional complexity of GISA is that one must propose exponents for the s-type basis functions.

The robustness of Hirshfeld-I (HI), ISA and GISA is compared in a computational assessment study. A set of 103 terminally blocked random penta-alanine conformers is optimized at the PBE0/6-311+G(d,p) level and the ground state density of each conformer is partitioned with the three schemes. This assessment reveals several robustness issues of the ISA scheme. 22 out of 103 ISA computations failed to converge and for the 81 successful cases, the convergence of ISA is an order of magnitude slower than GISA. The fluctuations of the atomic charges in the central residue over all conformers is used as a first measure for the lack of robustness. In this test, GISA is comparable to ISA, while the HI charges are up to a factor five less sensitive to conformational changes. Hence, our attempt to propose a more robust ISA variant is only partially successful: GISA always converges (in fewer iterations), but the conformational robustness has not improved.

The origins of the conformational sensitivity are further analyzed with a comparison of the con- 
tributions to the AIM population as a function of the distance from the nucleus. This comparison reveals that ISA and GISA charges are more sensitive to changes in density on neighboring atoms than to changes in density on the actual atom. This non-locality may lead to larger fluctuations on atomic charges, which are mostly caused by conformational changes and the polarization of neighboring atoms. The fluctuations of the HI AIMs are more localized for most atoms in penta-alanine, yet there are some exceptions. Given the excellent robustness of HI, this trend suggests that the locality of AIM density fluctuations is a sufficient (but not necessary) condition for robustness.

\section{Acknowledgments}

T.V., V.V.S. and M.W. were supported by the Fund for Scientific Research-Flanders (FWO), the Research Board of Ghent University (BOF), and BELSPO in the frame of IAP/6/27. Funding was also received from the European Research Council under the European Community's Seventh Framework Programme (FP7(2007-2013) ERC grant agreement no. 240483). The computational resources (Stevin Supercomputer Infrastructure) and services used in this work were provided by Ghent University. P.W.A. was supported by the Natural Sciences and Engineering Research Council of Canada and the Canada Research Chairs. The authors also thank Prof. P. Bultinck for the valuable and encouraging discusions.

[1] R. G. Parr, P. W. Ayers, R. F. Nalewajski, J. Phys. Chem. A 109 (2005) 3957-3959.

[2] C. F. Matta, R. F. W. Bader, J. Phys. Chem. A 110 (2006) 6365-6371.

[3] R. S. Mulliken, J. Chem. Phys. 23 (10) (1955) 1833-1840.

[4] P. Politzer, R. S. Mulliken, J. Chem. Phys. 55 (10) (1971) 5135-5136.

[5] F. De Proft, J. M. L. Martin, P. Geerlings, Chem. Phys. Lett. 250 (3-4) (1996) 393-401.

[6] T. Verstraelen, E. Pauwels, F. De Proft, V. Van Speybroeck, P. Geerlings, M. Waroquier, J. Chem. Theory Comput. 8 (2) (2011) 661-676.

[7] A. E. Reed, R. B. Weinstock, F. Weinhold, J. Chem. Phys. 83 (2) (1985) 735-746.

[8] R. F. W. Bader, Chem. Rev. 91 (5) (1991) 893-928.

[9] M. Devereux, P. L. A. Popelier, I. M. McLay, J. Comput. Chem. 30 (8) (2009) 1300-1318. 
[10] C. I. Bayly, P. Cieplak, W. Cornell, P. A. Kollman, J. Phys. Chem. 97 (40) (1993) 1026910280.

[11] P. Bultinck, C. Van Alsenoy, P. W. Ayers, R. C. Dorca, J. Chem. Phys. 126 (14) (2007) 144111.

[12] P. Bultinck, P. W. Ayers, S. Fias, K. Tiels, C. Van Alsenoy, Chem. Phys. Lett. 444 (1-3) (2007) 205-208.

[13] S. Catak, M. D’hooghe, T. Verstraelen, K. Hemelsoet, A. Van Nieuwenhove, H.-J. Ha, M. Waroquier, N. De Kimpe, V. Van Speybroeck, J. Org. Chem. 75 (13) (2010) 4530-4541.

[14] S. Van Damme, P. Bultinck, S. Fias, J. Chem. Theory Comput. 5 (2) (2009) 334-340.

[15] T. Verstraelen, V. Van Speybroeck, M. Waroquier, J. Chem. Phys. 131 (4) (2009) 044127.

[16] T. Verstraelen, S. V. Sukhomlinov, V. Van Speybroeck, M. Waroquier, K. S. Smirnov, J. Phys. Chem. C 116 (1) (2011) 490-504.

[17] T. C. Lillestolen, R. J. Wheatley, Chem. Commun. (45) (2008) 5909-5911.

[18] A. D. Becke, E. R. Johnson, A density-functional model of the dispersion interaction, J. Chem. Phys. 123 (15) (2005) 154101.

[19] A. Tkatchenko, M. Scheffler, Accurate molecular van der waals interactions from Ground-State electron density and Free-Atom reference data, Phys. Rev. Lett. 102 (7) (2009) 073005.

[20] S. N. Steinmann, C. Corminboeuf, A System-Dependent Density-Based dispersion correction, J. Chem. Theory Comput. 6 (7) (2010) 1990-2001.

[21] P. Bultinck, D. L. Cooper, D. Van Neck, Phys. Chem. Chem. Phys. 11 (18) (2009) 3424-3429.

[22] F. L. Hirshfeld, Theoret. Chem. Acc. 44 (2) (1977) 129-138.

[23] D. Vanfleteren, D. Van Neck, P. Bultinck, P. W. Ayers, M. Waroquier, Partitioning of the molecular density matrix over atoms and bonds, J. Chem. Phys. 132 (16) (2010) 164111.

[24] P. W. Ayers, J. Chem. Phys. 113 (24) (2000) 10886-10898.

[25] R. F. Nalewajski, R. G. Parr, Proc. Nat. Acad. Sci. (16) 8879-8882. 
[26] E. R. Davidson, S. Chakravorty, Theoret. Chem. Acc. 83 (5) (1992) 319-330.

[27] H. Weinstein, P. Politzer, S. Srebrenik, Theor. Chem. Acc. (2) 159-163.

[28] A. M. Simas, R. P. Sagar, A. C. T. Ku, V. H. Smith, Can. J. Chem. (8) 1923-1930.

[29] P. W. Ayers, R. G. Parr, Int. J. Quantum Chem. (6) 877-881.

[30] M. J. Frisch, G. W. Trucks, H. B. Schlegel, G. E. Scuseria, M. A. Robb, J. R. Cheeseman, G. Scalmani, V. Barone, B. Mennucci, G. A. Petersson, H. Nakatsuji, M. Caricato, X. Li, H. P. Hratchian, A. F. Izmaylov, J. Bloino, G. Zheng, J. L. Sonnenberg, M. Hada, M. Ehara, K. Toyota, R. Fukuda, J. Hasegawa, M. Ishida, T. Nakajima, Y. Honda, O. Kitao, H. Nakai, T. Vreven, J. A. Montgomery, Jr., J. E. Peralta, F. Ogliaro, M. Bearpark, J. J. Heyd, E. Brothers, K. N. Kudin, V. N. Staroverov, R. Kobayashi, J. Normand, K. Raghavachari, A. Rendell, J. C. Burant, S. S. Iyengar, J. Tomasi, M. Cossi, N. Rega, J. M. Millam, M. Klene, J. E. Knox, J. B. Cross, V. Bakken, C. Adamo, J. Jaramillo, R. Gomperts, R. E. Stratmann, O. Yazyev, A. J. Austin, R. Cammi, C. Pomelli, J. W. Ochterski, R. L. Martin, K. Morokuma, V. G. Zakrzewski, G. A. Voth, P. Salvador, J. J. Dannenberg, S. Dapprich, A. D. Daniels, O. Farkas, J. B. Foresman, J. V. Ortiz, J. Cioslowski, D. J. Fox, Gaussian 09 Revision A.02, Gaussian Inc. Wallingford CT, 2009.

[31] R. Krishnan, J. S. Binkley, R. Seeger, J. A. Pople, J. Chem. Phys. 72 (1) (1980) 650-654.

[32] M. J. Frisch, J. A. Pople, J. S. Binkley, J. Chem. Phys. 80 (7) (1984) 3265-3269.

[33] C. Adamo, V. Barone, J. Chem. Phys. 110 (13) (1999) 6158-6170.

[34] V. Lebedev, D. Laikov, Doklady Mathematics 59 (3) (1999) 477-481.

[35] T. C. Lillestolen, R. J. Wheatley, J. Chem. Phys. (14) 144101. 


\begin{tabular}{ccccc}
$k$ & $\mathrm{H}$ & $\mathrm{C}$ & $\mathrm{N}$ & $\mathrm{O}$ \\
\hline 1 & 5.672 & 148.3 & 178.0 & 220.1 \\
2 & 1.505 & 42.19 & 52.42 & 65.66 \\
3 & 0.5308 & 15.33 & 19.87 & 25.98 \\
4 & 0.2204 & 6.146 & 1.276 & 1.685 \\
5 & & 0.7846 & 0.6291 & 0.6860 \\
6 & & 0.2511 & 0.2857 & 0.2311
\end{tabular}

Table 1: The exponents for the Gaussian s-type density basis in the GISA method in atomic units. 


\begin{tabular}{cccccc} 
Atom & HI & ISA & GISA & QTAIM & RESP \\
\hline H:27 & 0.008 & 3.2 & 5.3 & 2.9 & 9.8 \\
H:29 & 0.008 & 1.6 & 2.1 & 1.9 & 2.9 \\
H:31 & 0.010 & 1.6 & 2.1 & 1.9 & 2.7 \\
C:28 & 0.009 & 4.1 & 2.4 & 1.2 & 8.6 \\
C:30 & 0.009 & 1.5 & 2.1 & 0.7 & 6.5 \\
C:34 & 0.010 & 4.3 & 3.8 & 1.1 & 12.3 \\
N:26 & 0.013 & 3.8 & 3.5 & 1.4 & 10.3 \\
O:35 & 0.017 & 1.2 & 1.2 & 0.7 & 4.5
\end{tabular}

Table 2: Comparison of the conformational fluctuations on AIM charges obtained with several schemes. The first columns contains the standard deviation of the Hirshfeld-I charges for some selected atoms (see Fig. 1). The other columns are standard deviations on AIM charges obtained with other schemes, divided by the corresponding value for the Hirshfeld-I scheme. 


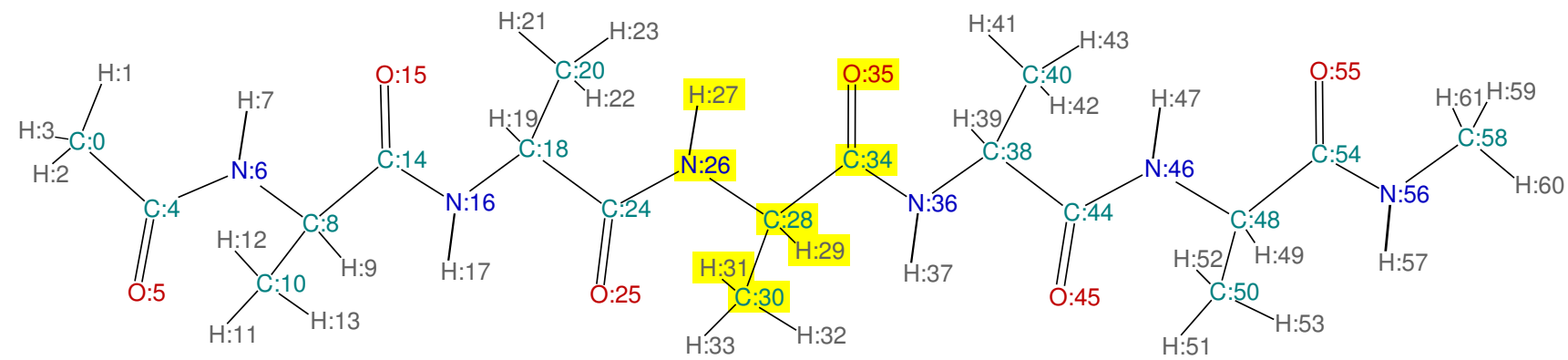

Figure 1: The terminally blocked penta-alanine structure with atomic indexes. The atoms selected for the analysis of the robustness are highlighted in yellow. 


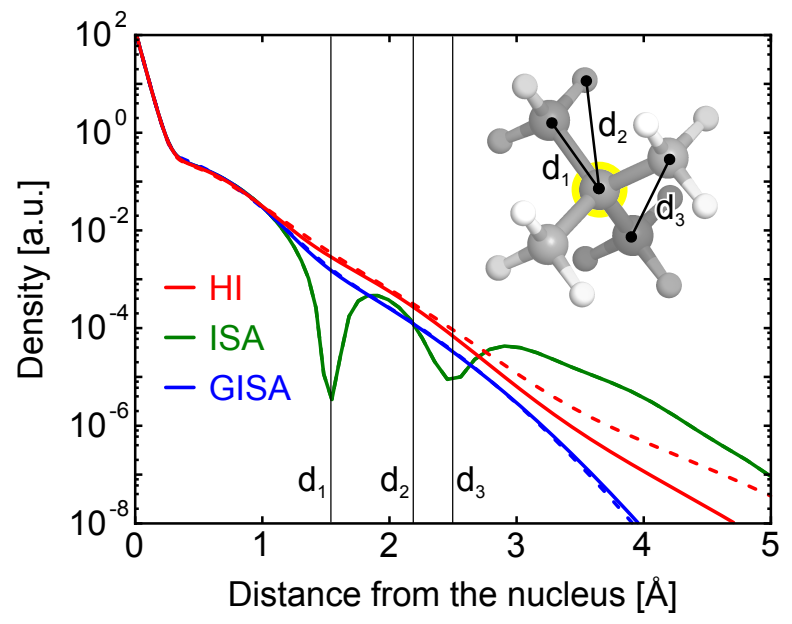

Figure 2: The pro-atomic density (dashed line) and the spherical AIM density (solid line) of the central carbon atom in neopentane at the PBE0/6-311+G(d,p) level of theory, as obtained with different stockholder partitioning schemes: red=HI, green=ISA, blue=GISA. The same color code is used in Figs. 3 and 4. The distance from the central carbon to the other carbon and hydrogen atoms are indicated as $d_{1}$ and $d_{2}$, respectively. The symbol $d_{3}$ is used for the distance between two methyl carbons. 

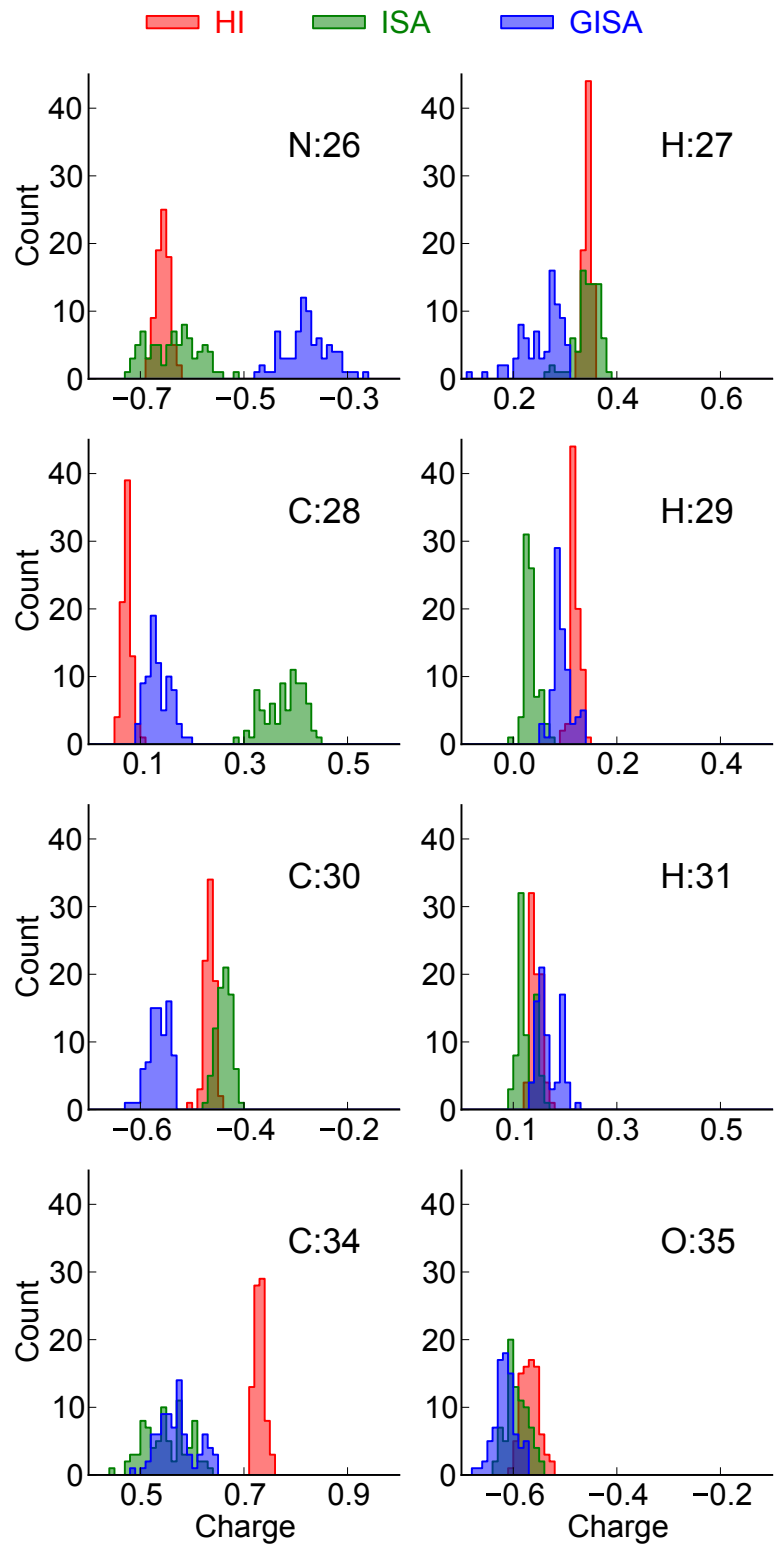

Figure 3: Histograms of the selected atomic charges (see Fig. 1) in the 81 penta-alanine conformers. To facilitate the comparison, the range of the $\mathrm{x}$-axis always spans $0.6 \mathrm{e}$. The same color code is used in Figs. 2 and 4 : red=HI, green $=\mathrm{ISA}$, blue $=$ GISA. 

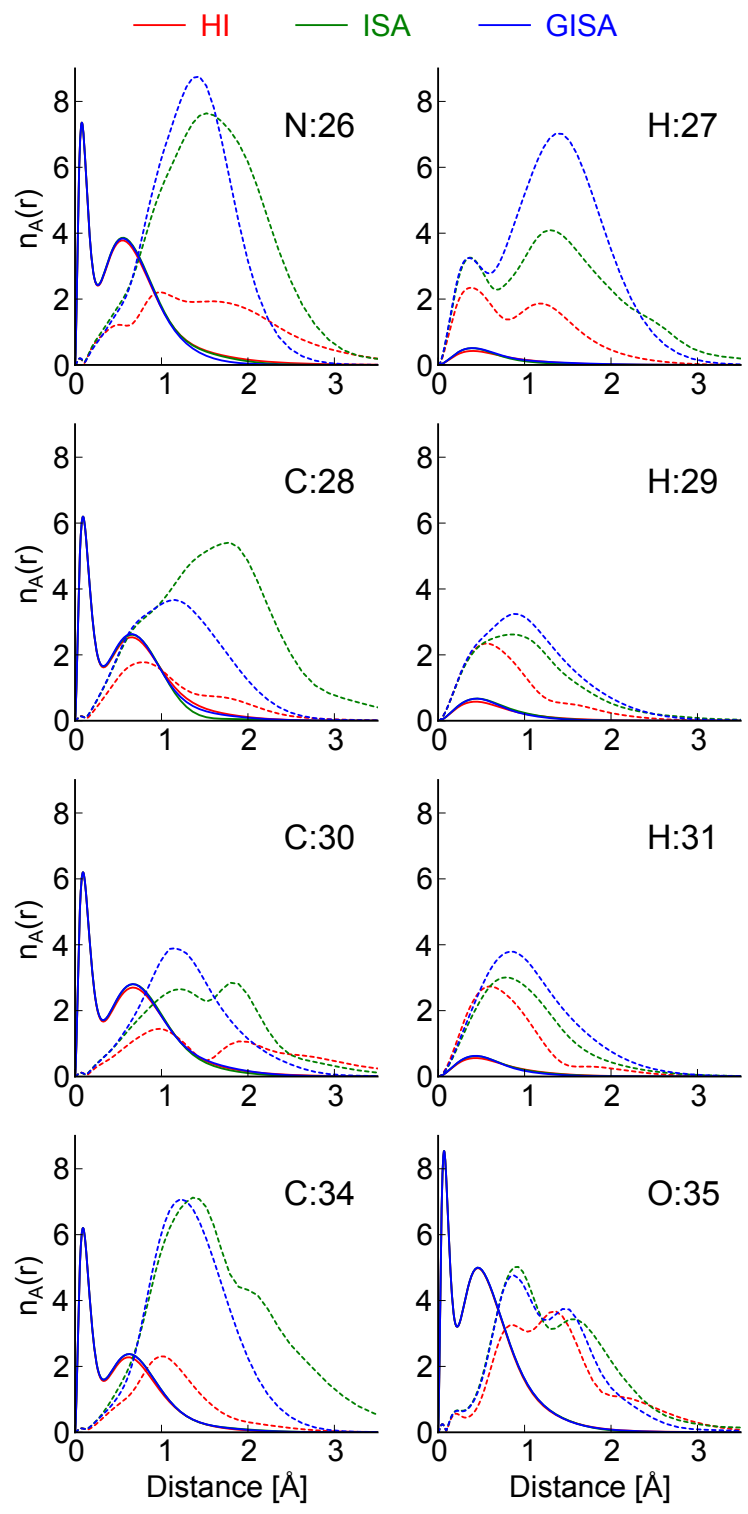

Figure 4: The average (solid) and the standard deviation times 400 (dashed) of the function $n_{A}(r)$ over the 81 penta-alanine conformers for a selection of atoms (see Fig. 1). The same color code is used in Figs. 2 and 3: red $=$ HI, green $=$ ISA, blue $=$ GISA. 